\title{
Acknowledgment to Reviewers of JMSE in 2020
}

\section{JMSE Editorial Office}

Citation: JMSE Editorial Office.

Acknowledgment to Reviewers of JMSE in 2020. J. Mar. Sci. Eng. 2021, 9, 110. https://doi.org/10.3390/ jmse 9020110

Published: 22 January 2021

Publisher's Note: MDPI stays neutral with regard to jurisdictional claims in published maps and institutional affiliations.

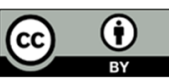

Copyright: (c) 2021 by the author. Licensee MDPI, Basel, Switzerland. This article is an open access article distributed under the terms and conditions of the Creative Commons Attribution (CC BY) license (http://creativecommons.org/licenses /by/4.0/).

MDPI AG, St. Alban-Anlage 66, 4052 Basel, Switzerland

Peer review is the driving force of journal development, and reviewers are gatekeepers who ensure that JMSE maintains its standards for the high quality of its published papers. Thanks to the cooperation of our reviewers, in 2020, the median time to first decision was 16 days and the median time to publication was 34 days. The editors would like to express their sincere gratitude to the following reviewers for their precious time and dedication, regardless of whether the papers were finally published:

Abaei, Mohammad Mahdi

Abascal, Ana J.

Abate, Giada

Abbas, Naseem

Abbasnia, Arash

Abdel-Maksoud, Moustafa

Abdullah, Nur Azam

Abed, Abdel Illah El

Abeysiriwardhane, Apsara

Abramović, Borna

Abramovich, Haim

Abramowicz-Gerigk, Teresa

Abrehdary, Majid

Acanfora, Maria

Accoroni, Stefano

Aciu, Claudiu

Acomi, Nicoleta

Adam, Frank

Adams, Aaron

Adamski, Mariusz Robert

Adcock, Christopher

Addona, Fabio

Adhikari, Ram

Afonso, Clélia Neves

Afonso, Fernando

Afshani, Alireza

Afshar-Mohajer, Nima

Aggarwal, Geetika

Agorreta, Ainhoa

Agrafiotis, Panagiotis

Aguirre Martinez, Gabriela Veronica

Aguzzi, Jacopo

Ahmadi, S. Farzad

Ahmadvand, Seyedsaeid

Ahn, Seongho
Aijaz, Saima

Ainslie, Michael

Akan, Cigdem

Akanji, Lateef

Akbar, Muhammad K.

Akib, Shatirah

Alam, Md Tauqeer

Alasali, Feras

Albano, Marco

Alberello, Alberto

Alberti, Marcos G.

Alborz, Niknam

Alcaide, Juan Ignacio

Alejo-Armijo, Alfonso

Alessandra, Saponieri

Ali, Muhammad

Alimonti, Claudio

Alizad, Karim

Alizadeh, Babak

Allahdadi, Mohammad Nabi

Almeida Ferreira, Hugo

Almeida Júnior, José Humberto

Alobaid, Falah

Alonso, Francisco

Alpers, Werner

Altenburger, Andreas

Altomare, Corrado

Alujević, Neven

Alvarez, Sergio

Alvarez-Palau, Eduard

Álvarez-Salgado, Xosé Antón

Alves, José Carlos

Amaechi, Chiemela Victor

Amini, Ali

Amini, Azin 
Ancellin, Matthieu

Andersen, Thomas Lykke

Anderson, David A.

Anderson, Ross

Andersson, Bengt

Ando, Keita

Andreev, Sergey

Andrei, Constantin-Octavian

Andrews, Charles

Andrianov, Igor

Andric, Jerolim

Andricevic, Roko

Andriolo, Umberto

Anfuso, Giorgio

Angeloudis, Athanasios

Anis, Ayal

Anton, Iulia

Antoniadou, Chryssanthi

Antonini, Alessandro

António Correia, José

Antunes Do Carmo, José Simão

Anzidei, Marco

Aoki, Shinichi

Aps, Robert

Aragonés, Luis

Araki, Susumu

Arashkevich, Elena G.

Araujo, Maria Amelia

Arbanas, Željko

Archetti, Renata

Arechavala-Lopez, Pablo

Arias, Andres

Arienzo, Michele

Armenio, Elvira

Arnaubec, Aurélien

Arndt, Erik

Arosio, Riccardo

Artal-Sevil, Jesús Sergio

Artero Guerrero, Jose Alfonso

Arula, Timo

Ashley, Burkett

Ateeq-ur-Rehman, Sardar

Atkinson, Michael

Atroshchenko, Elena

Atzori, Bruno

Augello, Riccardo

Aureli, Francesca

Austin, Martin

Avanzini, Giovanni Buizza

Awolayo, Adedapo

Ayca, Aykut
Ayed, Samah Ben

Azamathulla, Hazi M.

Azhikodan, Gubash

Azman, Lucija

Baba, Wassim Mohamed

Babonneau, Nathalie

Bacci, David

Bacci, Maurizio

Badger, Merete

Baek, Gahyun

Baek, Seung Ho

Bahamon, Nixon

Bahcevandziev, Kiril

Bai, Wei

Bailey, David

Bainbridge, Scott

Bakalis, Evangelos

Bakdi, Azzeddine

Baki, Abul BM

Baldauf, Michael

Balduzzi, Giuseppe

Balena, Pasquale

Balouin, Yann

Balsamo, Flavio

Balss, Ulrich

Baltzer, Agnès

Balzano, Sergio

Ban, Dario

Ban, Marko

Banerjee, Sourav

Banno, Masayuki

Baquerizo, Asunción

Baranowski, Paweł

Barbarelli, Silvio

Barbaro, Giuseppe

Barbieri, Roberto

Barbulescu, Alina

Bares, Pau

Baring, Ryan

Barinova, Sophia

Barker, Chris

Barmpatza, Alexandra C.

Barrett, Glyn A.

Barrineau, Patrick

Barton, Larry L.

Basha, Elizabeth

Bashir, Musa

Bašić, Josip

Basilone, Gualtiero

Battarra, Mattia

Bauer, Bernard O. 
Baxevani, Anastassia

Bayon, Yves

Becker, Dirk

Becking, Thomas

Bedon, Chiara

Beecraft, Laura

Beegle-Krause, Cynthia Juyne

Beemer, Ryan D.

Beirão, Pedro

Bejger, Artur

Belgiorno, Giacomo

Belibassakis, Kostas

Bell, Tom W.

Bellanova, Piero

Bellos, Evangelos

Belotti, Giorgio

Belotti, Roberto

Belu, Nadia

Belu, Radian

Ben Meftah, Mouldi

Benaouicha, Mustapha

Benassai, Guido

Benbouzid, Mohamed

Bencsik, Peter

Benetazzo, Alvise

Bengtsson, Mats

Benim, Ali Cemal

Beniuga, Razvan

Benjamins, Steven

Bennetts, Luke

Benoit, Michel

Bensi, Manuel

Benzerara, Karim

Berardi, Valentino Paolo

Bergheim, Asbjorn

Bergillos, Rafael J.

Bergström, Martin

Bermudez, Maria

Bernard, Guillaume

Bernardi Aubry, Fabrizio

Bernasconi, Giancarlo

Bernataviciene, Jolita

Bernhardt, Paul A.

Berni, Fabio

Berrue, Fabrice

Berta, Maristella

Bertin, Stéphane

Bertolo, Davide

Bertoni, Duccio

Bertorello, Carlo Francesco Mario

Besio, Giovanni
Besset, Manon

Bettencourt, João Paulo

Bezzi, Annelore

Bhalla, Amneet

Bharti, Vidhi

Bialik, Robert

Bielic, Toni

Bigerelle, Maxence

Bihs, Hans

Binda, Elisa

Bindzárová Gergel’ová, Marcela

Birk, Andreas

Birylo, Monika

Bismor, Dariusz

Biswas, Soumyajyoti

Bjarne, Landfald

Bjerklie, David M.

Björkqvist, Jan-Victor

Blagin, Evgeny

Blanco, Juan

Blanco-Chao, Ramon

Blanco-Fernandez, Pilar

Blažauskas, Nerijus

Blazejewski, Ryszard

Blazina, Maria

Blázquez, Mercedes

Blázquez-Salom, Macià

Blenkinsopp, Chris

Blokus, Agnieszka

Blondel, Philippe

Bobák, Martin

Bohl, Douglas G.

Böhm, Michał

Bokuniewicz, Henry

Bolbot, Victor

Bolognini, Luca

Bonamore, Alessandra

Bonar, Paul A. J.

Bonduà, Stefano

Bonin-Font, Francisco

Borbone, Nicola

Borges Vieira, Guilherme Bergmann

Borja, Angel

Borkowski, Piotr

Borrego-Díaz, Joaquin

Borsukiewicz-Gozdur, Aleksandra

Borthwick, Alistair

Borys, Andrzej

Bosa, Silvia

Bosma, Bret

Bošnjaković, Mladen 
Bosso, Luciano

Bot, Patrick

Botero, Camilo M.

Bothwell, John

Botwright, Natasha

Bou-cabo, Manuel

Boucheron, Romuald

Boudhane, Mohcine

Boulougouris, Evangelos

Bouvier, Clément

Bouzas, Angela Fontán

Bradshaw, Michael

Braginsky, Boris

Brajard, Julien

Brandt, Steven R.

Branger, Hubert

Braun, Burga

Bravo, Luis

Bravo, Teresa

Brcko, Tanja

Bregant, Luigi

Brennan, Anthony B.

Brenner, Steve

Brett, Per Olaf

Bricker, Jeremy D.

Briganti, Riccardo

Briggs, Tiffany

Brito, Moisés

Brizzolara, Stefano

Brkić, Dejan

Brkljač, Branko

Brocchini, Maurizio

Brogi, Simona Retelletti

Broszat, Dominik

Brown, Jenifer

Bruder, Brittany L.

Brundo, Maria Violetta

Brunone, Bruno

Brustolin, Marco C.

Bruzzone, Dario

Bryner, Elliott

Bubak, Iwona

Bubić, Tanja Šegvić

Bucci, Vittorio

Buccino, Mariano

Bucur, Daniel

Budzyńska, Agnieszka

Bujorianu, Luminita Manuela

Bukin, Oleg

Burdziakowski, Paweł

Burguera, Antoni
Burns, Alan

Buscaino, Giuseppa

Bushra, Nazla

Bustamante, Eliseo

Bustos, Alejandro

Butka, Brian

Buynevich, Ilya V.

Bystrowska, Marta

Caballes, Ciemon Frank

Cabezón, Rubén

Cabrera, Francisco

Cabrini, Marina

Cahill, Patrick L.

Calafat Frau, Antoni

Calafel, Itxaso

Calambokidis, John

Calcagnile, Lucio

Calcinai, Barbara

Caldeirinha, Vitor

Calisal, Sander

Camarero, Alberto

Cambazoglu, Kemal

Cambazoglu, Mustafa Kemal

Camiz, Sergio

Campbell, Anthony

Campisi, Tiziana

Campone, Luca

Campora, Ugo

Camus, Paula

Cañaveras, Juan Carlos

Candries, Maxim

Canepa, Monica

Cannata, Giovanni

Capello, Marco

Capone, Alessandro

Capra, Alessandro

Capurso, Tommaso

Carabin, Giovanni

Carbonara, Pierluigi

Cardona, Fabio

Čarija, Zoran

Carisi, Francesca

Carlson, Daniel F.

Carneiro, José Ricardo

Caroppo, Carmela

Carragher, John

Carral, Luis

Carrillo, José M.

Carvalho, Ana P.

Casado, Rafael

Casavola, Alessandro 
Caseiro Rocha, Alfredo Moreira

Castellani, Giulia

Castellino, Myrta

Catalin, Dumitrescu

Ćatipović, Ivan

Caulier, Guillaume

Cavagnaro, Robert J.

Cavallaro, Antonio

Cavallaro, Luca

Cavalli, Rosa

Cechowicz, Radosław

Cecioni, Claudia

Cecrdle, Jiri

Cedomir, Milosavlevic

Celli, Daniele

Cely, Juan S.

Cengel, Yunus

Ceran, Bartosz

Cerenius, Lage

Cerik, Burak Can

Cervi, Eric

Chabchoub, Amin

Chai, Wei

Chakraborty, Shamik

Chakraborty, Sudip

Chakraborty, Sumit

Chalikov, Dmitry

Chan, Benny Kwok Kan

Chan, I-Chi

Chan, Shu Ning

Chandar, Dominic

Chandra, Alvin

Chandrala, Lakshmana

Chang, Ching-Chih

Chang, Daejun

Chang, Hsien-Kuo

Chang, Hsuan

Chang, Shuchih Ernest

Chang, Yeon S.

Chang, Yu-Chia

Chang, Yu-Shan

Chatzikokolakis, Konstantinos

Chau, Kwok-Wing

Chaussonnet, Geoffroy

Che, Jiaxing

Chelazzi, David

Chella, Mayilvahanan Alagan

Chen, Bang-Fuh

Chen, Baoshan

Chen, Jun

Chen, Qiang
Chen, $\mathrm{Ru}$

Chen, Wei-Bo

Cheng, Jun

Cheng, Yang

Cheng, Zhen

Chenuil, Anne

Cheriton, Olivia

Chernin, Leon

Chernov, Ilya A.

Chiaradonna, Anna

Chickadel, Chris

Chirico, Giovanni

Chiu, Yung-Chia

Cho, Byunggu

Cho, Hong Yeon

Cho, Hyun Jung

Cho, Ik-Soon

Cho, Nak-Kyun

Choi, Hyeung-sik

Choi, Jungho

Chondros, Michalis K

Chow, Aaron C.

Chow, Yi-Chih

Christie, Simon

Christodoulou, Anastasia

Christou, Epaminondas D.

Chu, Chia-Chu

Chung, Jaewoo

Chwała, Marcin

Chybicki, Andrzej

Chybowski, Leszek

Ciappa, Achille

Cima, Francesca

Cipollini, Francesca

Clavero, Maria

Clinci, Catalin

Cloquell-Ballester, Víctor

Clott, Christopher

Coccossis, Harry

Cococcioni, Marco

Cohn, Nicholas

Coiro, Domenico P.

Coker, Phil

Colantoni, Andrea

Coleman, Shirley

Collareta, Alberto

Conides, Alexis

Conley, Daniel

Contardo, Stephanie

Contestabile, Pasquale

Contreras-López, Manuel 
Convertino, Matteo

Copping, Andrea E.

Ćorak, Maro

Corigliano, Pasqualino

Corn, Marko

Cornwell, Jeffrey

Correia, Anacleto

Correia, José A.F.O.

Cortese, Barbara

Corvaro, Sara

Cosoli, Simone

Costa, Pedro J.M.

Couzinet-Mossion, Aurelie

Cozijn, Hans

Cozzoli, Francesco

Cremades Ugarte, Javier

Crise, Alessandro

Critchley, Alan T.

Cronin, Meghan F.

Cucco, Andrea

Cui, Linlin

Cui, Yong

Čulin, Jelena

Culloch, Ross M.

Cunha, Pedro

Cutroneo, Laura

Cuttler, Michael V. W.

Cyganek, Bogusław

Cyr, Frederic

Czapik, Przemysław

Czaplewski, Krzysztof

Czarnota, Robert

Czechowski, Leszek

Czermański, Ernest

D'Agostino, Paul

D'Alessandro, Felice

Dagang, Zhao

Dahl, Peter

Dai, Jian

Dai, Jiguo

Dai, Saishuai

Daidzic, Nihad

Dailianis, Thanos

Dairain, Annabelle

Dalaklis, Dimitrios

Daley, Claude

Damacharla, Praveen

Damiani, Leonardo

Dan, Sebastian

Danacova, Michaela

Danezis, Chris
Daniele, Casalbore

Danijela, Bogner

Danklmayer, Andreas

Dankowski, Hendrik

Danner, Tobias

Darmon, Michel

Darsena, Donatella

Das Neves, Luciana

Dashtimanesh, Abbas

Dastgheib, Ali

Datta, Rahul

Davey, Thomas A. D.

Davidson, Josh

Davies, Ian

Davis, R. Benjamin

Dawes, Warrick R.

Dawid, Leszek

Dawson, Heather A.

De Aguiar, Claudinei Rodrigues

De Camargo, Felipe Vannucchi

De Dios, J.Carlos

De Finis, Rosa

De La Morena, Joaquin

De Luca, Fabio

De Macedo, Carina Regina

De Oliveira, Mario Anderson

De Pace, Francesco

De Rosa, Alessandro

De Sanjosé Blasco, José Juan

De Santi, Francesca

De Serio, Francesca

Degago, Samson A.

Degiuli, Nastia

Degtyarev, Alexander

Dejhalla, Roko

Del Campo, Jose Maria

Deleersnijder, Eric

Delgado-Fernandez, Irene

Delis, Anargiros

Demarq, Hervé

Demirbilek, Zeki

Demková, Lenka

Deng, Yun

Denissenko, Petr

Denner, Fabian

Dennington, Simon

Dentale, Fabio

Dev, Arun

Dhaliwal, Gurpinder Singh

Dhariwal, Rohit

Dharmarathne, Suranga 
Di Blasio, Gabriele

Di Bona, Gianpaolo

Di Camillo, Crsitina Gioia

Di Ciaccio, Fabiana

Di Fiore, Vincenzo

Di Francesco, Silvia

Di Paola, Gianluigi

Di Risio, Marcello

Di Sarli, Valeria

Di Vaio, Assunta

Diaconescu, Mihail

Diaz, Rafael

Díaz-Carrasco, Pilar

Diaz-Hernandez, Gabriel

Dierick, Noël

Diermanse, Ferdinand L. M.

Dietrich, Daniel

Dimas, Athanassios

Dinis, Pedro

Disimile, Peter J.

Djidjeli, Kamal

Djordjevic, Nenad

Do Carmo, Ricardo

Dolgikh, Grigory Ivanovich

Dombek, Grzegorz

Dominey-Howes, Dale

Domínguez, Herminia

Dominguez, Sergio

Domínguez-Pérez, Dany

Domitrović, Josipa

Donadio, Carlo

Donaire, Alejandro

Dondajewska-Pielka, Renata

Dong, Shuchuang

Dong, Yuepeng

Dornbusch, Uwe

Doujak, Eduard

Dounas, Costas

Dragan, Valeriu

Draycott, Samuel

Driggers, William B.

Drillet, Yann

Dubaj, Tibor

Dubbioso, Giulio

Dubrovsky, Victor Anatoly

Dufour, Suzanne

Dulebenets, Maxim A.

Dunn, Ryan

Duplančić Leder, Tea

Dupont, Sam

Durán-Riveroll, Lorena
Durbin, Paul

Durland, Evan

Duru, Okan

Dussin, Raphael

Dutour-Sikiric, Mathieu

Dutta, Parikshit

Dworak, Paweł

Dyrcz, Czesław

Dziula, Przemyslaw

Dzolkarnaini, Nazam

Eames, Ian

Earthy, Jonathan

Echevarría, Fidel

Echevin, Vincent

Edesess, Ariel J.

Edge, Billy

Egea, Antonio

Ehlers, Sören

Eiksund, Gudmund Reidar

Eitzinger, Christian

Ekergård, Boel

Ekins, Merrick

El Kacimi, Younes

Elibol, Armagan

Eliopoulou, Eleftheria

Elken, Jüri

Ellenson, Ashley

Elsaesser, Bjoern

El-Shafee, Omar Osama

Emblemsvåg, Jan

Emil, Bayramov

Enalou, Hossein Balaghi

Engler, Evelin

Ensig-Karup, Alan

Erikson, Li

Escobar, Yesid Carvajal

Eslam-Panah, Azar

Esnaola, Ganix

Esselborn, Saskia

Esteban, Maria Dolores

Estevadeordal, Jordi

Etemaddar, Mahmoud

Etemad-Shahidi, A.

Eugenio González, Francisco

Eugenio, Francisco

Evelpidou, Niki

Evin, Emil

Evrard, Fabien

Ewans, Kevin

Exadactylos, Athanasios

Ezer, Tal 
Ezzeddine, Wajih

Fabrizio, Pistani

Facca, Chiara

Facevicova, Kamila

Faedo, Nicolás

Faganeli, Jadran

Failla, Giuseppe

Fairall, Christopher W.

Fairley, Iain

Faivre, Sanja

Falcao, Antonio

Falcieri, Francesco

Falkenberg, Laura

Falkowska, Ewa

Fan, Songmiao

Fanini, Lucia

Faraci, Carla

Farcas, Adrian

Farid, Muhammad Shahid

Farina, Almo

Farkas, Andrea

Farshchi Yazdi, Seyed Amir Fouad

Fattah, Islam Md Rizwanul

Favali, Paolo

Favaretto, Chiara

Fei, Tai

Feil, Roland

Felbeck, Horst

Felski, Andrzej

Feng, Hui

Feng, Jie

Ferdani, Daniele

Fernandes, Célio

Fernández De Puelles, M Luz

Fernández-Isabel, Alberto

Fernandez-Nunez, Miriam

Ferrara, Giuseppe

Ferrari, Francesco

Ferrari, Silvia

Ferraro, Luciana

Ferreira, Bruno

Ferreira, Fausto

Ferreira, José Carlos

Ferris, Timothy

Ferson, Scott

Figari, Massimo

Figueira, William

Figuero, Andrés

Filgueira-Vizoso, Almudena

Filianoti, Pasquale G. F.

Filin, Sagi
Filina-Dawidowicz, Ludmiła

Filippas, Evangelos

Fingas, Merv

Fiorenza, Roberto

Firth, Callum R.

Fischer, Peter

Fisher, Alexander

Fissel, David B.

Fitzsimmons, Patrick

Flick, Reinhard E.

Florin, Pacuraru

Fogla, Navin

Fonseca, Nuno

Fontan Bouzas, Angela

Fontanarosa, Donato

Forest, Josep

Formentin, Sara Mizar

Forster, Ian

Fortes, Juana

Foteinos, Michael I.

Foti, Enrico

Fouatih, Omar Madani

Fournier, Georges

Fox-Hughes, Paul

Fragassa, Cristiano

Fraile-Nuez, Eugenio

Frančić, Vlado

Francone, Antonio

Frankovska, Jana

Franz, Guilherme

Franzitta, Vincenzo

Fraser, Timothy

Frau, Diego G.

Frédéric, Muttin

Freeman, Justin

Frega, Ferdinando

French-McCay, Deborah P.

Friedland, René

Friedrichs, Carl T.

Frigaard, Peter

Frost, Carwyn

Fuentes-Pérez, Juan Francisco

Fuhrman, David

Fujiwara, Toshifumi

Fulton, Beth

Furue, Ryo

Gabl, Roman

Gaeta, Maria Gabriella

Galati, Antonino

Galeazzi, Roberto

Gallach-Sánchez, David 
Gallerano, Francesco

Gallet, Sebastien

Galyaev, Andrey

Gama, Cristina

Gamito, Sofia

Ganju, Neil

Ganske, Anette

Gantsevich, Mikhail

Gao, Xinghua

Gao, Yan

Garbaras, Andrius

Garbowska, Monika

García Espinosa, Julio

Garcia Navarro, Pigar

García, Juan T.

García, Marcos R.

Garcia-Alonso, Lorena

García-Marín, José Luis

García-Romero, Leví

Garza-Pérez, Joaquin Rodrigo

Garzon, Juan L.

Gastineau, Romain

Gatti, Filippo

Gattuso, Domenico

Gavrilov, Milivoj B.

Gebreegziabher, Merhawi

Gedikli, Ersegun Deniz

Geert Hiddink, Jan

Geiger, Gerhard

Gendron, Paul

Geng, Zongyu

Gerber, Doron

Gerber, Stefan

Gertman, Isaac

Ghaemi, Hossein

Ghoreyshi, Seyed Mohammad

Giallanza, Antonio

Giangrande, Adriana

Giannini, Gianmaria

Gibbons, Steven J.

Gil-Alana, Luis Alberiko

Gilbert, James M.

Gilja, Gordon

Giorgio, Trincas

Girolami, Laurence

Girovský, Peter

Gish, Lynn Andrew

Giuseppe, Arcangeli

Gkerekos, Christos

Głuchowski, Andrzej

Glynn, Peter J.
Gnatowski, Andrzej

Goda, Ibrahim

Goes, Marlos

Goethals, Peter

Gohari, Soheil

Goiran, Jean-Philippe

Gold-Bouchot, Gerardo

Goldfinger, Chris

Golewski, Grzegorz Ludwik

Golias, Mihalis

Golimowski, Wojciech

Gollasch, Stephan

Gomari, Sina Rezaei

Gomes, Rui P. F.

Gomez Cerezo, Maria Natividad

Gomez Gesteira, Moncho

Gómez, Fernando

Gomez-Pau, Alvaro

Gomez-Pujol, Lluis

Gonçalves, Ana M. M.

Gonçalves-Araujo, Rafael

Goncharov, Alexey

Gong, Jiawei

González-Villanueva, Rita

Goreau, Thomas J. F.

Goring, Derek

Górniak, Aleksander

Górski, Jarosław

Górski, Krzysztof

Göteman, Malin

Goto, Satoru

Goudarzy, Meisam

Grabe, Jürgen

Grabowski, Martha

Gracia Prieto, Francisco Javier

Grant, Jonathan

Greco, Michele

Grémare, Antoine

Griggs, Gary

Grigorieva, Vika G.

Grigoropoulos, Gregory

Grilli, Gaetano

Grimstad, Gustav

Grm, Aleksander

Groenenboom, Paul

Groll, Nikolaus

Groothuis, Peter

Gros, Jonas

Gryning, Sven-Erik

Grzadziela, Andrzej

Grznár, Patrik 
Gualeni, Paola

Gualtieri, Carlo

Guan, Pai-Chen

Gucma, Lucjan

Gucma, Maciej

Gucwa, Marek

Gudino-Elizondo, Napoleon

Gudmestad, Ove Tobias

Guillou, Nicolas

Gumulya, Monica

Gundić, Ana

Gusev, Anatoly

Gutekunst, Kirstin

Gutiérrez-Romero, José E.

Gutow, Lars

Guze, Sambor

Guzzini, Alessandro

Habel, Shellie

Hace, Aleš

Haces-Fernandez, Francisco

Häfele, Jan

Haghdoust, Pouya

Hahn, Axel

Haider, Julfikar

Hajime, Kayanne

Hajivalie, Fatemeh

Hala, David

Hallegraeff, Gustaaf M.

Hallerman, Eric

Hallowell, Spencer

Hally, David

Halse, Karl Henning

Hamad, Rebeen Ali

Hamawand, Ihsan

Hamilton, Peter

Hämmerling, Mateusz

Han, Manbae

Hanaoka, Shinya

Haneklaus, Nils

Haney, J. Christopher

Hanif, Asad

Hannan, Mohammed Abdul

Hansen, Anne Merrild

Happel, Austin

Hara, Yutaka

Harada, Naomi

Harig, Sven

Harirchian, Ehsan

Harlaß, Jan

Harmelin, Jean-George

Harris, Larry
Haselsteiner, Andreas

Hashimoto, Hirotada

Hass, H. Christian

Hassan, Marwa

Hassanaly, Malik

Hatziioannou, Marianthi

Hay Mele, Bruno

Hayes, Daniel

Hazelwood, Richard A.

$\mathrm{He}$, Bao-Jie

Hegermiller, Christie A.

Heidarpour Shahrezaei, Iman

Heidarzadeh, Mohammad

Heinrich, Martin

Held, Philipp

Heller, Valentin

Hellio, Claire

Helmons, Rudy

Hemer, Mark

Hemery, Lenaig

Hemmati, Arman

Hendrickson, Kelli

Henesey, Lawrence E.

Henkelmann, Bernhard

Henryk, Lasota

Heo, Man-Woong

Hequette, Arnaud

Herdzik, Jerzy

Hering-Bertram, Martin

Hernandez Sosa, Daniel

Hernández Sosa, José Daniel

Herrero, Elías Revestido

Herreros López, Alberto

Herrling, Gerald

Herrmann, Frank

Herterich, James

Heshmati-alamdari, Shahab

Hiraishi, Tetsuya

Hirdaris, Spyros E.

Hirose, Yuu

Hlaváčová, Irena M.

Ho, Ching-Hsien

Ho, Long

Hoang, Nguyen Tien

Hoecker-Martinez, Martin

Hoeksema, Bert

Hoenders, Bernhard J.

Hoffmann, Norbert

Hojan, Marcin

Hole, Lars Robert

Hong, Sung-Min 


Honkonen, Tuula
Honório, Leonardo De Mello
Horecka, Beata
Hotea, Ionela
Hoth, Julian
Hou, Qingzhi
Houser, Chris
Howe, John A.
Howell, Richard M.
Hristov, Tihomir
Hrustić, Enis
Hsiao, Shih-Chun
Hsiao, Sung-Shan
Hsieh, Chi-Ming
Hsu, Hsien-Pin
Hsu, Hung-Chu
Hu, Aixue
Hu, Fei
Huang, Haosheng
Huang, Hsin-Haou
Huang, Jong-Chin
Huang, Shaoguang
Huang, Ting-Hsuan
Huang, Tse-Hung
Huang, Wei
Hübler, Clemens
Hulatt, Christopher J.
Huntley, Helga
Huybrechts, Nicolas
Hwang, Hun-Gyu
Hynninen, Antti
Iatridi, Zacharoula
Ichikawa, Kaoru
Idier, Déborah
Ielciu, Irina
Iglesias, Gregorio
Igoe, David
Iijima, Kazuhiro
Iiyama, Masaaki
Iizuka, Satoshi
Ilies, Nicoleta
Inazu, Daisuke
Inhestern, Lukas Benjamin
Inoue, Hirofumi
Ínsua, Álvaro Baaliña
Inturri, Giuseppe
Ioannis Vyrides, Ioannis
Ioannis, Hatzianestis
Iosub, Marina
Iqbal, Hafiz M. N.
IRIE, Takahiro

Honkonen, Tuula

Honório, Leonardo De Mello

Horecka, Beata

Hotea, Ionela

Hoth, Julian

Hou, Qingzhi

Houser, Chris

Howe, John A.

Howell, Richard M.

Hristov, Tihomir

Hrustić, Enis

Hsiao, Shih-Chun

Hsiao, Sung-Shan

Hsieh, Chi-Ming

Hsu, Hsien-Pin

Hsu, Hung-Chu

$\mathrm{Hu}$, Aixue

$\mathrm{Hu}, \mathrm{Fei}$

Huang, Haosheng

Huang, Hsin-Haou

Huang, Jong-Chin

Huang, Shaoguang

Huang, Ting-Hsuan

Huang, Tse-Hung

Huang, Wei

Hübler, Clemens

Hulatt, Christopher J.

Huntley, Helga

Huybrechts, Nicolas

Hwang, Hun-Gyu

Ichikawa, Kaoru

Idier, Déborah

lelciu, Irina

Iglesias, Gregorio

Igoe, David

Iijima, Kazuhiro

Iiyama, Masaaki

Iizuka, Satoshi

Ilies, Nicoleta

Inazu, Daisuke

Inhestern, Lukas Benjamin

Inoue, Hirofum

Ínsua, Álvaro Baaliña

Inturri, Giuseppe

Ioannis Vyrides, Ioannis

Ioannis, Hatzianestis

Iqbal, Hafiz M. N.

IRIE, Takahiro
Irwin, Andrew J.

Ishii, Daisuke

Isla, Federico

Istrati, Denis

Itsukushima, Rei

Iuppa, Claudio

Ivanić, Maja

Ivić, Stefan

Iwanowicz, Luke R.

Izquierdo, Alfredo

Izquierdo, Juan

Izquierdo, Miguel

Izumi, Mitsuru

Jaffe, Jules

Jagrič, Vita

Jajac, Nikša

Jakobsen, Ken-Robert G.

Jakobsson, Antti

Jaksic, Vesna

Jalbi, Saleh

Jamieson, Alan

Jan, Chyan-Long

Janowski, Lukasz

Jasim, Abbas F.

Jasionowski, Robert

Jastrzębska, Małgorzata

Javier, Carlos

Je, drasik, Jan

Jefferson, Thomas A.

Jehel, Pierre

Jeng, Dong-Sheng

Jeong, Jungsik

Jeong, Seong-Yeob

Jesus, Joaquim

Jeung, In-Seuck

Jia, Boru

Jiang, Danchi

Jiang, Long

Jiang, Ruinian

Jimenez, Carlos

Jiménez-Herranz, José Miguel

Jimeno-Saez, Patricia

Jin, Chungkuk

Jin, Yaqing

Jo, Sung-woong

Johansson, Maria T.

Johnson, Erick

Johnson, Kent C.

Johnson, Markes

Johnstone, Cameron

Jóia Santos, Catarina 
Jolliff, Jason K.

Jonathan, Philip

Jones, Kevin

Jong-In, Byun

Jorge Luis Zambrano, Martinez

Jovanovic, Jelena

$\mathrm{Ju}$, Shen-haw

$\mathrm{Ju}, \mathrm{Yu}-\mathrm{Min}$

Juez, Carmelo

Jurkovic, Martin

Justino, Paulo

Kaczmarek, Halina

Kadivar, Ebrahim

Kafel, Alina

Kagemoto, Hiroshi

Kaidi, Sami

Kalinić, Hrvoje

Kalra, Tarandeep Singh

Kam, Moshe

Kamalanathan, Manoj

Kamath, Arun

Kambezidis, Harry D.

Kamranzad, Bahareh

Kang, Chang-Keun

Kang, Sungwook

Kang, Xinyi

Kao, Sheng-Long

Kapalavavi, Brahmam

Kaplunenko, Dmitry

Kapustin, Ivan A.

Kara, Kursat

Karabil, Sitar

Karagiannis, Nikos

Karahalios, Hristos

Karakasidis, Theodoros

Karambas, Theophanis V.

Karampour, Hassan

Karathanasi, Flora

Karimi, Hamid Reza

Karki, Sita

Karkoschka, Erich

Karoui-Yaakoub, Narjess

Karozis, Stelios

Karpowicz, Maciej

Kastrinaki, Georgia

Kasumyan, Alexander

Katalinic, Marko

Katopodes, Nikolaos

Katsiropoulos, Christos

Katunin, Andrzej

Kaup, Magdalena
Kauppi, Laura

Kaur, Aman

Kaushik, Swati

Kazemifar, Farzan

Kazmierczak, Andrzej R.

Kazuo, Konagai

Kechagias-Stamatis, Odysseas

Keller, Aimee

Kelman, Candice Carr

Kelpšaitè-Rimkiené, Loreta

Kelsey, R. Heath

Kempf, Philipp

Kenji, Toyota

Kępniak, Maja

Keramaris, Evangelos

Keshavan, Jishnu

Khabbaz, Hadi

Khait, Anatoliy

Kharitonov, Dmitry

Khayyer, Abbas

Khazaeinejad, Payam

Khodadadian, Amirreza

Khovanov, Igor

Khurshid, Shahid

Kiguchi, Masashi

Kilby, Jeff

Kilcher, Levi

Kilibarda, Zoran

Kim, Chang-Bae

Kim, Dokyun

Kim, Dongkyu

Kim, Ekaterina

Kim, Hyeon-ju

Kim, Hyun-won

Kim, Jonghoek

Kim, Kyeong Ok

Kim, Kyu-Han

Kim, Seungjun

Kim, Sooyoul

Kim, Youngbok

Kim, Youngjoo

Kingon, Kelly

Kinnaird, Tim

Kinnas, Spyros A.

Kirchner, Stefan

Kishore, Ravi Anant

Kitaguchi, Hirotaka

Kitazawa, Daisuke

Kitsiou, Dimitra

Kjersem, Kristina

Klenke, Thomas 
Klimach, Harald

Klonaris, Georgios

Knieling, Jörg

Knigge, Thomas

Kobetičová, Klára

Koch, Reinhard

Kodikara, Jayantha

Kok, Annebelle

Kokinou, Eleni

Koksharova, Olga A.

Kolakowski, Zbigniew

Kolander, Robert

Kolendowicz, Leszek

Kolerski, Tomasz

Koll, Katinka

Kombiadou, Katerina

Kong, Xiangxiong

Konispoliatis, Dimitrios

Konovessis, Dimitrios

Kontakiotis, George

Kontoni, Denise-Penelope N.

Kontopoulos, Ioannis

Kontovas, Christos

Kooistra, Wiebe H. C. F.

Kopelevich, Oleg

Kõrgesaar, Mihkel

Kornev, Nikolai

Korobiichuk, Igor

Koskeridou, Efterpi

Kosturek, Robert

Koszelew, Jolanta

Kotrasová, Kamila

Kotwicki, Lech

Koubogiannis, Dimitrios

Kouchaki, Samaneh

Kouhi, Soroush

Koushan, Kourosh

Kovács, János

Kowalczewska-Madura, Katarzyna

Kowalewska-Kalkowska, Halina

Kramer, Stephan

Kranjčević, Lado

Krasuski, Kamil

Krčum, Maja

Kress, Nurit

Krestenitis, Yannis N.

Krieger, Michael

Krinkin, Kirill

Krishna PIllai, Karthika

Křivý, Vit

Kroeger, Silke
Kruml, Tomáš

Kruschel, Claudia

Krvavica, Nino

Krystosik-Gromadzinska, Agata

$\mathrm{Ku}$, Namkug

Kubit, Andrzej

Kudasik, Mateusz

Kuibin, Pavel Anatolievich

Kujala, Pentti

Kuklinski, Piotr

Kulich, Miroslav

Kumar, Dipankar

Kumar, Praveen

Kuo, Ting-Chun

Kurevija, Tomislav

Kurkina, Oxana E.

Kurushina, Victoria

Kuszewski, Hubert

Kuwahara, Takuya

Kuznetsov, Sergey

Kuznetsova, Alexandra

Kvittem, Marit Irene

Kwak, Moon K.

Kwak, Seonyeong

Kwiecien, Joanna

Kwong, Kevin B.

Kyozuka, Yusaku

Kyun Kim, Do

Kyvelou, Stella Sofia

Labbozzetta, Manuela

Labeur, Robert Jan

Łabuz, Tomasz

Lackovic, Igor

Lader, Pål Furset

Laface, Valentina

Lafforgue, Michel

Łagoda, Tadeusz

Lai, Chun-Ta

Lake, Thomas

Lamas Galdo, María Isabel

Lamas, María Isabel

Landsberger, Sheldon

Lane-Serff, Gregory

Lanzafame, Rosario

Larson, Erik B.

Lasserre, Frederic

Lau, Yui-yip

Laureano-Rosario, Abdiel E.

Lauria, Agostino

Lavado, Ramon

Lavidas, George 
Laviola, Michele

Lazar, Michael

Łazuga, Kinga

Lazzeri, Luigi

Le Heron, Richard Bernard

Le, T. T. Yen

Le, Tuyen Quang

Le, Viet Hung

Leach, Felix

Lea-Smith, David

Lebeau, Juliana

Lebedevas, Sergejus

LeBlanc, James

Leblond, Jeff

Leduc, Damien

Lee, Chang Jun

Lee, Changhee

Lee, Dong Eun

Lee, Han Soo

Lee, Hee-Keun

Lee, Hsien Hua

Lee, Jung Sun

Lee, Kenneth

Lee, Keunhwa

Lee, Kevin M.

Lee, Meng-Tsung

Lee, Se-Jin

Lee, Tim

Lee, Tsu-Chuan

Lee, Woo-Dong

Lefèvre, Nathalie

Legg, Sonya

Lehr, Bill

Lehtoranta, Kati

Lei, Po-Ruey (Barry)

Lekkala, M.K.Reddy

Lemmer, Frank

Lemnitzer, Anne

Lemu, Hirpa G.

Lenain, Luc

Leniowska, Lucyna

Lenoble, Veronique

Leopardi, Angelo

Lepadatu, Daniel

Lesen, Amy E.

Levacher, Daniel

Lewińska, Paulina

Lewis, Helen

Lewis, Matthew

Li, Chengxi

Li, Fang
Li, Honghai

Li, Hua

Li, Jianghui

Li, Ji-Hong

Li, Tie

Li, Yuzhu

Liang, Hui

Liberzon, Dan

Libric, Lovorka

Lidtke, Artur K

Lie, Arne

Lieber, Lilian

Liedermann, Marcel Georg

Ligęza, Paweł

Ligi, Martin

Ligteringen, Han

Liguori, Francesco

Lim, Youngsub

Lin, Chang-Sheng

Lin, Chia-Hua

Lin, Hanzhi

Lin, Lee-Yaw

Lin, Meng-Yu

Lin, Senjie

Lin, Ting-Chieh

Lin, Yu-Hsien

Lind, Pedro G.

Lindøe, Preben Hempel

Lipa, Belinda

Lippert, Tristan

Lira-Loarca, Andrea

Lisowski, Edward

Litwin, Wojciech

Liu, Haoran

Liu, Jinjie

Liu, Jinming

Liu, Shukui

Liu, Wen-Cheng

Liu, Yingyi

Liu, Yonggang

Liu, Yucheng

Liu, Zhaowei

Liu, Zhuo

Ljubešić, Zrinka

Llano-Serna, Marcelo

Lo Giudice, Angelina

Loh, Andrew

Loh, Hui Shan

Lomonaco, Pedro

Loncar, Goran

Lopes, Carina L. 
Lopes, José Fortes

Lopes, Miguel

Lopez, Guiomar

López-Jiménez, P. Amparo

Lopez-Querol, Susana

López-Sanmartín, Monserrat

Loskot, Pavel

Lotfian, Saeid

Low, Eicher

$\mathrm{Lu}$, Biao

Lu, Fu-I

Lucas, Donald

Luciani, Valeria

Luigi, Mucerino

Luijendijk, Arjen

Lund, Ivar

Lungu, Adrian

Lunkov, Andrey

Luo, Bingkun

Luo, Hongliang

Luo, Min

Luque-Nieto, Miguel Angel

Lušić, Zvonimir

Lusito, Letizia

Lützen, Marie

Lyridis, Dimitrios V.

Lyszkowicz, Adam

Lyu, Wenjing

M'zoughi, Fares

Mabon, Leslie J.

Macali, Armando

Macfarlane, Gregor

MacGregor, Barbara J.

Machicoane, Nathanael

MacKinnon, Scott

Madurapperuma, Buddhika

Magalhães, Leandro

Magalhães, Solange

Magg, Sven

Maglić, Lovro

Mahmoudi Kurdistani, Sahameddin

Mahmoudian, Nina

Majak, Jüri

Majumder, Sudip

Makar, Artur

Makarov, Denis V.

Makris, Christos

Malara, Giovanni

Malcangio, Daniela

Malecha, Ziemowit

Mallios, Angelos
Malvic, Tomislov

Man, Yemao

Mancini, Simone

Mancuso, Antonio

Manenti, Sauro

Manger, Walter L.

Manheim, Frank T.

Maniscalco, Rosanna

Mańkowska, Marta

Mannarini, Gianandrea

Manzano-Agugliaro, Francisco

Manzetti, Sergio Otterlei

Marchel, Łukasz

Marcos, Rubal

Margaritelli, Giulia

Margolis, Donald L.

Maria Giuseppina Chiara, Nestola

Marin, Andrea

Marin, Maria Gabriella

Marinkovic, Dragan

Marinò, Alberto

Marino, Enzo

Marino, Giada

Marino, Massimiliano

Marino, Raffaele

Marinov, Marin

Mariusz, Domagala

Marseglia, Guido

Martí, José Vicente

Martin, Karin

Martín, Manuel

Martin, Ruth A.

Martinát, Stanislav

Martinelli, Luca

Martinez, Rodrigo

Martinez, Xavier

Martínez-Espinosa, Rosa María

Martini, Alberto

Martins, Alfredo Manuel De Oliveira

Martins, Fabio J. W. A.

Martins, Kévin

Martins, Marcos

Martins, Marcos Silva

Martins, Roberto

Maruschak, Pavlo

Maruyama, Shigenori

Masajada, Jan

Mascioli, Francesco

Masek, Vlastimil

Mason, Robert

Maspataud, Aurélie 
Massot-Campos, Miquel Francisco

Mastbergen, Dick R.

Mastronuzzi, Giuseppe

Masumoto, Yukio

Mata-Lima, Herlander

Mateescu, Carmen

Matic, Frano

Matsuba, Yoshinao

Matsuo, Kaoru

Matsushita, Yoshiki

Matt, Tranter

Mattiazzo, Giuliana

Matulja, Tin

Mauro, Francesco

Mavrakos, Spyros A.

Maximov, Alexey

Mayer, Bernhard

Mayo, Talea

Maza, Maria

Mazarakos, Thomas P.

Maze, Guillaume

Mazerbourg, Sabine

Mazzoleni, Maurizio

McAllister, Mark

McCay, Deborah French

McCormack, Grace

McDougal, Owen M.

McDowell, Judith E.

McGuinness, Justin P.L.

McLaughlin, Shawn

McNaughton, James

McPhaden, Michael James

Medeiros, Stephen C.

Mehrabi, Armin

Meisen, Tobias

Mejia, Max

Mel, Riccardo

Memos, Constantine D.

Mendes, Mário C.

Mendes, Susana

Mendonça, Paulo

Mendoza-Porras, Omar

Meneguz, Marco

Menga, Nicola

Menke, William

Mentaschi, Lorenzo

Menza, Charles

Mercorelli, Paolo

Mestanza-Ramón, Carlos

Mestres, Marc

Meylan, Michael H.
Mèžinė, Jovita

Miccadei, Enrico

Michailides, Constantine

Michalak, Izabela

Michalet, Richard

Michel, Guillaume

Michele, Simone

Mickeviciene, Rima

Mierzwiński, Dariusz

Migliaccio, Maurizio

Mikkelsen, Ove

Mikkola, Tommi Kristian

Milad, Benmadi

Milad, Ferdowsi

Milbrandt, Eric

Min, Rui

Minh, Le Van

Minoura, Munehiko

Minzoni, Rebecca Totten

Miot Da Silva, Graziela

Mir, Zahid Mohammad

Mirauda, Domenica

Miszczyk, Andrzej

Mizutani, Norimi

Mleneck-Vautravers, Maryline

Młynarczyk, Przemysław

Mobley, Curtis

Mochida, Kazuhiko

Moeini, Ghazal

Mofidi, Amir

Mohammed Geba, Khaled

Mohapatra, Sarat

Mohović, Đani

Molfetta, Matteo Gianluca

Molina, Rafael

Molines Llodrá, Jorge

Molkov, Alexander

Molyneux, David

Monieta, Jan

Moñino, Antonio

Montblanc, Tomas Fernandez

Moon, Hyeonjoon

Morales Maqueda, Miguel Ángel

Moreira, Lúcia

Moreno, Luis

Moreno-Gutiérrez, Juan

Mori, Masaaki

Moro, Lorenzo

Morris, Rebecca

Morsilli, Michele

Mortlock, Thomas 
Morton, Steve L.

Moshou, Alexandra

Mostafa, Ahmed

Motta, Chiara Maria

Mottershead, Derek

Mottola, Fabio

Mouga, Teresa

Moura Paredes, Guilherme

Mousaviraad, Maysam

Moyano, Francisco

Mrówczyńska, Maria

Mrozik, Karol Dawid

Mrzljak, Vedran

Muglia, Mike

Mukherjee, Rajib

Munehiko, Hinatsu

Murai, Motohiko

Muralidharan, Balaji

Murea, Cornel Marius

Murray, Rory O'Hara

Murray, Thomas

Murzin, Serguei Petrovich

Musumeci, Rosaria Ester

Mutsuda, Hidemi

Muttin, Frédéric

Naar, David

Nagata, Takayuki

Nagel, Tim

Nagle-McNaughton, Timothy

Najafi, Mohammad

Nakagawa, Yasuyuki

Nakamura, Ryota

Nakamura, Tomoaki

Nakata, Haruhiko

Nam, Bo

Nam, Sang-Jip

Nam, SungHyun

Nam, Woo-chul

Naman, C. Benjamin

Nanjappa, Deepak

Nanjundaswamy, Ananda

Naseri, Masoud

Näslund, Johan

Nattkemper, Tim W.

Naus, Krzysztof

Nava, Vincenzo

Navarro, Gabriel

Navrátil, Václav

Nazeer, Majid

Neary, Vincent

Nebot, Enrique
Nedjai, Rachid

Nędzarek, Arkadiusz

Nejad, Amir R.

Neshat, Mehdi

Netto, Theodoro A.

Neumann, Thomas

Nevshupa, Roman A

Ngo, Son Ich

Nguyen, Phong T. T.

Nguyen, Trieu

Nichols, C. Reid

Nicolae, Florin

Nielsen, Kim

Nielsen, Ulrik Dam

Nihei, Yasunori

Nijland, Wiebe

Nikitas, Georgios

Nikolaidis, Alexandros K.

Nikolaos Pagonis, Dimitrios

Nikolay, Rogovtsov

Nikolic, Dejan

Nikou, Alexandros

Nishi, Yoshiki

Nivedita, Nivedita

Noble, Donald

Noblesse, Francis

Noghabaei, Mojtaba

Noh, Yoojeong

Nordam, Tor

Norman, Rosemary

Notti, Emilio

Novelli, Guillaume

Nowaczyk, Jacek

Ntislidou, Chrysoula

Nunes, Leonel Jorge Ribeiro

Núñez, Luis Ramón

Núñez-Pons, Laura

Nunn, Patrick

Nuortila, Carolin

Nuuttila, Hanna Katariina

Nyka, Maciej

Nysten-Haarala, Soili

O'Byrne, Michael

O'Mahony, Micheál

O'Shea, Michael

Oakley, Brian A.

Obelcz, Jeffrey B.

Obulisamy, Parthiba Karthikeyan

Oday Ibraheem, Abdullah

Odetti, Angelo

Ogashawara, Igor 
Oh, Daekyun

Oh, Won Chun

Ohtsuka, Satoru

Okamoto, Daniel K.

Olaniyi, Eunice Omolola

Oleinik, Phelype Haron

Olejnik, Bartosz

Olejnik, Paweł

Olejníková, Petra

Oliveira, Dinis

Oliveto, Giuseppe

Olli, Kalle

Omer, Joshua

Omerdic, Edin

Onas, Adrian S.

Onea, Florin

ONeill, Andrea

Oniszczuk-Jastrząbek, Aneta

Ono, Kenji

Ono, Tsuneo

Onuma, Takeshi A.

Oriňáková, Renáta

Ortega, José Marcos

Ortega-Sánchez, Miguel

Ortiz, Francisco

Ortiz-Suslow, David

Orzech, Mark D.

Osborne, Al

Oshima, Nobuyuki

Osipova, Liudmila

Osório, Jonas H.

Ostnes, Runar

Ostrowski, Rafał

Oterkus, Erkan

Oterkus, Selda

Otero, Pablo

Otremba, Zbigniew

Ottogalli, Kiara

Ouahsine, Abdellatif

Øvergård, Kjell Ivar

Owens, Katharine A.

Pabis, Krzysztof

Paduan, Jeff

Padula, Matthew P.

Pagliaroli, Alessandro

Pahwa, Anil

Paik, Kwang-Jun

Painter, Stuart C.

Painting, Suzanne

Pająk, Michał

Palleschi, Federica
Pallikaris, Athanasios

Palumbo, Annunziata

Pamuković, Jelena Kilić

Pan, Yang

Pan, Yulin

Panagakos, George

Panagoulia, Dionysia

Panciroli, Riccardo

Pandit, Ravi

Pandkar, Anup

Pannone, Marilena

Paolo, Favali

Papadaki, Maria

Papadimitriou, Kimon

Papaefthimiou, Spiros

Papathanasiou, Theodosios K.

Papathoma-Köhle, Maria

Papenmeier, Svenja

Paprota, Maciej

Parfen'ev, Vladimir M.

Parham, Kiyan

Pariotis, Efthimios

Paris, Enio

Paris, Paul J.

Park, Changhyup

Park, Deuk-Jin

Park, Hyoungsu

Park, Joo Myun

Park, Joseph

Park, Mincheol

Park, Nam Kyu

Park, Sae Woong

Park, Sang Kil

Park, Sewan

Park, Soo Je

Park, Su-hyun

Park, Sung-Sik

Park, Sunho

Park, Woo-Sun

Park, Youngsoo

Parra Santiago, Jose Ignacio

Parra, Lorena

Parsons, Michael

Parunov, Josko

Pasanisi, Francesco

Pascal, Rémy Claude René

Pascarella, Domenico

Pascolo, Sara

Pasha, Junayed

Pasquali, Davide

Passow, Uta 
Pastorino, Paolo

Pastusiak, Tadeusz

Patsch, Kiki

Paul, Satyam

Paul, Shiladitya

Paula, José Ricardo

Paulauskas, Vytautas

Payo, Andres

Payo-Payo, Ana

Paz Montelongo, Soraya

Paz, Igor

Pazouki, Kayvan

Peakman, Aiden

Pech, Martin

Pegau, Scott

Pekour, Mikhail

Pelinovsky, Efim

Peller, Julie

Pelletier, Emilien

Pempkowiak, Janusz

Peña González, Enrique

Penalba, Markel

Peng, Heather

Peng, Jie

Peng, Ming-Tsan

Peng, Yan-Tsung

Penna, Nadia

Pennino, Silvia

Pepe, Fabrizio

Peperzak, Louis

Peppa, Sofia

Perales Perales, Jose Manuel

Pereira, Filipe S.

Pereira, Leonel

Pérez Arribas, Francisco

Perez Gladish, Blanca

Pérez Rojas, Luis

Perez-Collazo, Carlos

Pérez-Sánchez, Modesto

Pergent, Gérard

Perkovic, Marko

Perna, Massimo

Perrone, Pierluigi

Perš, Janez

Pervizpour, Mesut

Pesch, Alexander $\mathrm{H}$.

Petcu, Cristian

Petersen, Mark

Petersen, Øyvind Wiig

Peterson, Kara J.

Pethiyagoda, Ravindra
Petrakis, Evangelos

Petrov, Pavel

Petrova, Daniela

Peyrard, Christophe

Pfouga, Alain

Phan, Hoang-Phuong

Philipp, Mucha

Phillips, Ross Owen

Piechna, Janusz

Pierella, Fabio

Pierre, Pouzet

Pierri, Cataldo

Pietrafesa, Leonard J.

Pikelj, Kristina

Pilch, Adam

Piloto, Paulo

Pinardi, Monica

Pinault, Jean-Louis

Pineda-Sanchez, Manuel

Pires-Silva, António A.

Piscopo, Vincenzo

Piskozub, Jacek

Piskur, Paweł

Píštěk, Václav

Piwosz, Kasia

Plech, Anton

Plomaritis, Theocharis A

Plotnikov, Leonid

Poguluri, Sunny Kumar

Poizot, Emmanuel

Politis, Gerasimos

Poljak, Igor

Pollock, Michael M.

Polonsky, Alexander

Polyansky, Oleg P.

Pombo, Ana

Ponce De León, Sonia

Ponta, Linda

Porathe, Thomas

Porowska, Dorota

Posey, Martin H.

Postacchini, Matteo

Postolache, Florin

Potaniec, Bartłomiej

Potemra, James

Poulain, Pierre-Marie

Poulicek, Mathieu E.A.

Poulis, Hans

Povilanskas, Ramūnas

Prachař, Aleš

Pranzini, Enzo 
Prapotnik Brdnik, Anita

Premnath, Kannan N.

Prendergast, Luke J.

Prevoroski, Zdenek

Price, James F.

Pringle, William J.

Prins, Theo

Pritz, Balazs

Proença Brójo, Francisco Miguel Ribeiro

Prospathopoulos, Aristides

Provencher, Jennifer F.

Prunet, Patrick

Psaraftis, Harilaos N.

Pugliese Carratelli, Eugenio

Pujades, Estanislao

Qi, Chongchong

Qi, Guangyan

Qi, Junyu

Qian, Qin

Quaranta, Franco

Quarta, Gianluca

Quattrocchi, Giovanni

Quddus, Noor

Quetzalcóatl, Omar Gutierrez

Quevedo, Eduardo

Rabalais, Nancy N.

Rabczuk, Timon

Radchenko, Mykola

Radenovic, Stojan

Radicella, Sandro

Radicioni, Fabio

Radnejad, Amir Bahman

Raghavan, Nagarajan

Raghukumar, Kaustubha

Rahman, Azizur

Rahman, M. Azizur

Rahman, Mizanur

Rahman, Mohammad A

Rahman, Mostafiz

Rahman, Saydur

Raileanu, Alina Beatrice

Raineault, Nicole

Raiteri, Giancarlo

Rajendran, Parvathy

Rajfur, Małgorzata

Rakotomamonjy, Thomas

Rakowska, Aleksandra

Ralston, Emily

Ram, Angia Sriram Pradeep

Raman-Nair, Wayne

Rampino, Michael Robert
Randazzo, Giovanni

Rapalis, Paulius

Raspanti, Mario

Rasskazov, Ilia

Ratilal, Purnima

Rauffet, Philippe

Raul Marin, Eng

Ravens, Thomas M.

Ray, Tathagata

Raykova, Reneta

Reale, Ferdinando

Rebelo, Ana Cristina

Rebelo, Carlos

Regan, Fiona

Rego, Francisco

Rehel, Karine

Reinap, Avo

Reinecke, Robert

Relich, Marcin

Rende, Sante Francesco

Rene, Eldon

Repolho, Tiago

Reynolds, Laura

Rezaei Gomari, Sina

Rezk, Hegazy

Rialland, Agathe Isabelle

Ribeiro, Américo S.

Ribeiro, Eraldo

Ribeiro, Sergio Silva

Ribeiro, Tiago

Ricchi, Antonio

Ricci, Alessio

Ricci, Stefano

Richiedei, Dario

Richter, Kristin

Ridolfi, Alessandro

Riley, James

Rinaldi, Giovanni

Ringwood, John

Risk, Michael J

Riska, Kaj Antero

Ritos, Konstantinos

Rizvi, Md Jahir

Rizvi, Zarghaam Haider

Rizzo, Carmen

Rizzo, Piervincenzo

Robert, Katleen

Roberty, Stephane

Robin, Nicolas

Roche, Marc

Rodella, Ilaria 
Rodrigues, Andreia C. M.

Rodrigues, Frederico

Rodrigues, José Alberto

Rodrigues, José Miguel

Rodrigues, Marta

Rodrigues, Vinícius Picanço

Rodríguez, Orlando Camargo

Rodriguez-Perez, Daniel

Rodriguez-Rodriguez, Javier

Roeber, Volker

Roffer, Mitchell

Rogan Šmuc, Nastja

Roggenbuck, Ole

Rogowski, Krzysztof

Rogowski, Peter

Romano, Alessandro

Romano, Stefania

Romanoff, Jani

Romero, Diego

Roméro-Ramirez, Alicia

Romolo, Alessandra

Roncin, Kostia

Ronconi, Robert

Ronen, Shuki

Rontani, Jean-François

Rosano, Gennaro

Rosas, Jose H. Ablanedo

Rossi, Riccardo

Rossi, Veronica

Rößler, Sebastian

Rostami, Masoud

Rouseff, Daniel

Roy, Spandan

Różyński, Grzegorz

Ruberti, Daniela

Rubio, Fernando

Ruesink, Jennifer

Ruessink, Gerben

Ruey-Syan, Shih

Ruggeri, Paolo

Ruggieri, Rosario

Ruiz-Sinoga, José Damián

Ruju, Andrea

Rundo, Massimo

Ruol, Piero

Ruponen, Pekka

Rurek, Mirosław

Rusu, Eugen

Rusu, Liliana

Rybak, Jarosław

Rybarczyk, Dominik
Rychert, Krzysztof

Rydbeck, Adam

Ryu, Keun

Ryu, Sungmin

Rządkowski, Romuald

Rzepkowska, Malgorzata

Rzhanov, Yuri

S. Silva, Simone

Sabaliauskas, Tomas

Sabau, Gabriela

Sadeghigoghari, Moslem

Sadowski, Maciej

Saffari Pour, Mohsen

Saghi, Hassan

Saharidis, Georgios

Sahoo, Prasanta

Saidi, Neila

Saint-Paul, Ulrich

Sajid, Muhammad

Sakamoto, Makaki

Sakamoto, Nobuaki

Sakata, Masahiro

Salauddin, Md

Salmun, Haydee

Salta, Maria

Salvadore, Francesco

Salvetti, Ovidio

Samanta, Dhrubajyoti

Samaras, Achilleas G.

Sampaio, Ana

Sampath, Dissanayake

Sampath, Kaushik

Sampson, Helen

Sánchez-Fernández, Manuel

Sankaran, Ganapathy Subramanian

Sanna, Laura

Sano, Masaaki

Sansò, Paolo

Santana Rodríguez, Juan José

Santana, Angelo

Santos, Angela

Santos, José Maria

Santos, Paulo

Santos, Rita B.

Sanuy Vazquez, Marc

Saponieri, Alessandra

Saprykina, Yana

Saqib, Muhammad

Sara, Pensieri

Sarda, Edoardo

Sardari, Roya R R 
Sarkar, Saptarshi

Sarti, Giovanni

Sartini, Ludovica

Sasa, Kenji

Sasaki, Noriyuki

Sassa, Shinji

Satoh, Masaki

Satoh, Yuhi

Sattar, Farook

Saviano, Simona

Savin, Eric

Savoca, Serena

Sawaragi, Tetsuo

Sawicki, Jerzy

Sayed, Khairy

Saylam, Kutalmis

Scamardella, Antonio

Scandura, Pietro

Scaradozzi, David

Scheffers, Anja

Scheffers, Sander

Schendel, Alexander

Scheres, Babette

Schimmels, Stefan

Schinas, Orestis

Schlembach, Florian

Schmid, Moritz S.

Schmitt, Pal

Schmitt, Thierry

Schneider Von Deimling, Jens

Schoefs, Franck

Schubert, Nadine

Scrano, Laura

Scurtu, Ionut Cristian

Semedo, Alvaro

Semenov, Yuriy

Seo, Byung-Kuk

Seo, Jeonghwa

Seo, Jung Kwan

Seo, Yutaek

Seok, Jun

Seong, Woo-Jae

Sepúlveda Allende, Andrés

Serani, Andrea

Sergeeva, Valentina M.

Sergievskaya, I.

Seroka, Greg

Serra, Teresa

Service, Matthew

Seyffert, Harleigh

Sezen, Savas
Sfravara, Felice

Shaeri, Saeed

Shafahi, Maryam

Shalby, Mohammad

Shand, Tom

Sharov, Andrey

Shehu, Rafael

Shemer, Lev

Shen, Chao

Shen, Jian

Shepard, Samuel

Sheremet, Alex

Shi, Fengyan

Shi, Lei

Shibasaki, Ryuichi

Shih, Dong-Sin

Shikov, Alexander N.

Shin, Seungho

Shin, Sung-chul

Shin, Sungwon

Short, Andrew

Shugan, Igor

Shukla, Dhwanil

Siddiqui, Mohd Atif

Siddiqui, Salman I.

Sidoti, David

Siedliska, Anna

Siegel, Stefan

Sieni, Elisabetta

Sieradzki, Rafał

Silva, Filipe

Silva, Paulo Alves

Silva, Sérgio Ribeiro E

Silveira, Graça

Silveira, Tanya

Simeonov, Julian

Simha, Ashutosh

Simian, Dana

Simon, Márta

Simpson, Matthew J. R.

Sinama, Frantz

Singh, Yogang

Sinsabvarodom, Chana

Sitaraman, Hariswaran

Sivanesan, Iyyakkannu

Sivasubramanian, Jayahar

Skriptsova, Anna V.

Slapnicar, Vedran

Slišković, Merica

Smith, Jane McKee

Smith, Travis A. 
Smoleń, Andrzej

Smułek, Wojciech

So, Christopher R.

Soares, Amílcar

Soares, Carlos Guedes

Sobieraj-Żłobińska, Anna

Sobolev, Dmitri

Socas-Rodríguez, Bárbara

Soda, Josko

Søiland, Henrik

Sokolovskiy, Mikhail A.

Solandt, Jean-Luc

Solazzi, Luigi

Solheim Pettersen, Sigurd

Sołtysik, Maciej

Somayajula, Abhilash

Somov, Yevgeny

Son, Min

Sondak, David

Song, Soonseok

Song, Yuhe Tony

Soo, Kim Hyun

Soria Garcia, Juan Miguel

Sorte, Sandra

Sotner, Roman

Sottolichio, Aldo

Souppez, Jean-Baptiste R. G.

Sousa, Vânia Serrão

Spagnoli, Andrea

Spagnoli, Federico

Spagnoli, Giovanni

Spasojevic, Pavle

Spaulding, Malcolm

Specht, Cezary

Specht, Mariusz

Spiridon, Stefan-Ionut

Splinter, Kristen

Spyrou, Kostas J.

Sserwadda, Martin

Stacul, Stefano

Stagonas, Dimitris

Stamatis, Nikolaos

Staninska-Pięta, Justyna

Stanivuk, Tatjana

Stanko, Milan

Stanley, Geoff

Stansby, Peter K.

Staszak, Maciej

Stateczny, Andrzej

Stavroulakis, Georgios E.

Stefanakos, Christos
Stephens, Thomas

Stergiopoulos, George

Stevenson, Emily

Steynor, Jeffrey

Stockenreiter, Maria

Stöcker, Claudia

Stolle, Jacob

Stone, Mike

Stonik, Valentin

Strehlow, Brian

Streicher, Maximilian

Strungaru, Stefan-Adrian

Strušnik, Dušan

Stuhlmeier, Raphael

$\mathrm{Su}$, Hongbo

Su, Nan-Jay

$\mathrm{Su}, \mathrm{Ruoyu}$

$\mathrm{Su}$, Tsung-chow

$\mathrm{Su}$, Wu-Chung

Suanez, Serge

Suara, Kabir

Subban, Chinmayee

Subramaniam, Aravinth

Subramanian, Rahul

Sudakov, Ivan

Suslov, Sergey

Swain, Geoffrey

Swayze, Gregg A.

Świt, Grzegorz

Sylaiou, Stella

Szabo, Lorand

Szafranek-Nakonieczna, Anna

Szatten, Dawid

Sze, Eric Tung-Po

Sziebig, Gabor

Szmańda, Jacek

Szostakiewicz-Hołownia, Marzena

Szpica, Dariusz

Szwajka, Krzysztof

Szypcio, Zenon

Tabeta, Shigeru

Taborda, Rui

Tafone, Alessio

Tagg, Alexander

Tagliafierro, Bonaventura

Tajima, Yoshimitsu

Takabatake, Tomoyuki

Takagi, Hiroshi

Talei, Amin

Tallini, Marco

Tam, Ivan CK 
Tambroni, Nicoletta

Tamizdoust, Mohammadreza Mir

Tampucci, Marco

Tan, Danielle S.

Tanaka, Hitoshi

Tanaka, Yuki

Tang, Hansong

Tang, Hung-Jie

Tang, Lijun

Taniuchi, Yukiko

Tankiewicz, Matylda

Taravella, Brandon M.

Tarnapowicz, Dariusz

Taskar, Bhushan

Tavokoli, Sasan

Tay, Zhi Yung

Taylor, Andy

Teague, Jonathan

Tedesco, Giacomo

Teimourian, Amir

Tekes, Ayse

Telegdi, Judit

Teletchea, Fabrice

Temiz, Irina

Tenekedjiev, Kiril

Teodoriu, Catalin

Terefenko, Paweł

Terêncio, Daniela

Terziev, Momchil

Tester, Patricia A.

Tetu, Amélie

Težak, Denis

Tezdogan, Tahsin

Themelis, Nikos

Theodoropoulos, Christos

Theotokatos, Gerasimos

Thepsithar, Prapisala

Thiébaut, Maxime

Thomsen, Jonas Bjerg

Thomsen, Laurenz

Thornton, Blair

Thresher, Robert

Thun, Kristian

Tian, Fang-Bao

Timko, Patrick G.

Tinh, Nguyen Xuan

Tlidi, Mustapha

Todaro, Francesco

Tolkova, Elena

Tomasicchio, Giuseppe Roberto

Tomaszewski, Dariusz
Tomoyoshi, Nishimura

Toniolo, Horacio

Tõnisson, Hannes

Torikai, Hiroyuki

Tornabene, Francesco

Tornatore, Cinzia

Török, Gergely T.

Torres, Ricardo

Tortosa, Dídac D.

Totti, Cecilia

Toyoma, Tadashi

Tran Anh, Duong

Traving, Sachia

Tregua, Marco

Trevlakis, Stylianos E.

Triay-Portella, Raül

Trimble, Sarah

Trimulyono, Andi

Trump, Benjamin D.

Tsanakas, Konstantinos

Tsao, Che-Chih

Tsau, Jyun-Syung

Tsekouras, G. J.

Tsoukala, Vasiliki K.

Tsujimoto, Masaru

Tsujimura, Taku

Tucci, Fausto

Tudela, Jose

Tufa, Ramato Ashu

Turetta, Alessio

Tutak, Magdalena

Tymokha, Oleksandr

Tzabiras, George D.

Tziavos, Nikolaos

Tzovenis, Ioannis

Ueno, Mikinori

Ujević, Ivana

Ulazia, Alain

Ullman, David S.

Unger, Shem

Urbaniak, Maciej

Ushida, Akiomi

Ustun, Taha Selim

Uttieri, Marco

Uzunoglu, Emre

Vafeidis, Athanasios

Vafidis, Dimitris

Vahtmäe, Ele

Valcic, Marko

Valčić, Sanjin

Vale, Carlos Alberto Garcia Do 
Vale, Paulo

Valente, Alessio

Valentine, Kendall

Vali, Zahra

Valipour, Mohammad

Vallée, Till

Valyrakis, Manousos

Van Den Burg, Sander W. K.

Van Der Heijden, G. H. M.

Van Der Meer, Jenstje

Van Der Reijden, K. J.

Van Der Werf, Jebbe

Van Hoydonck, Wim

Van Oppen, Madeleine

Van Rij, Jennifer

Van Steenberge, Maarten

Van Straalen, Nico M.

Van Walree, Frans

Vanden-Broeck, Jean-Marc

Varadinov, Maria

Varbanov, Petar

Varriale, Luisa

Vasconcelos, Helena Cristina

Vasileiadou, Katerina

Vasquez, Adrian A.

Vásquez, Julio A.

Vassalos, Dracos

Vaughn, Derrick

Vaz, Nuno

Vedyagin, Aleksey A.

Vega, Luis A.

Veldman, Arthur E. P.

Velicu, Radu

Velikanov, Vladimir

Vemulapalli, Vidyasiri

Ventura, Manuel

Veremey, Evgeny

Verhagen, Henk Jan

Verhelst, Sebastian

Verma, Amrit Shankar

Vernengo, Giuliano

Vervaeke, William C.

Verwaest, Toon

Vicinanza, Diego

Vidal, Carlos

Vidal, César

Vieira, Vasco M. N. C. S.

Viennois, Gaëlle

Vigevano, Luigi

Viggor, Signe

Vilarrasa, Victor
Vilibic, Ivica

Villa, Diego

Villani, M.

Viman, Liviu

Vinh, Nguyen Quang

Vinnem, Jan-Erik

Viso, Richard

Vitiello, Luigi

Vittori, Giovanna

Viveiros, Carla

Vladimir, Nikola

Vlahostergios, Zinon

Vlaskos, Ioannis

Von Lukas, Uwe Freiherr

Vos, Sander

$\mathrm{Vu}$, Mai The

Vuillaume, Jean-Francois

Vujović, Igor

Vukelic, Goran

Waagbo, Rune

Wadman, Heidi

Wagner, Beatrice

Wagner, Natalia

Wain, Danielle

Wakabayashi, Nobukazu

Waldman, Christoph

Wall Bell, Carrie C.

Wan, Ling

Wang, Bohong

Wang, Chi-Jo

Wang, Haibin

Wang, He-sheg

Wang, Jiaze

Wang, Jindong

Wang, Kun

Wang, Mian C.

Wang, Shan

Wang, Shaofeng

Wang, Taiping

Wang, Weizhi

Wang, Xin

Wang, Yongxiang

Wang, Youjiang

Warmann, Emily

Warmink, Jord J.

Wartzok, Douglas

Wase, Nishikant

Wasilewski, Marek

Wassermann, Jérôme

Watanabe, Yasushi

Watter, Holger 
Wawrzyniak, Natalia

Wcislik, Miroslaw

Wei, Yong

Weinbauer, Markus

Weintrit, Adam

Weiss, Taylor

Weisse, Ralf

Wellens, Peter

Weng, Wen-Kai

Westbury, Michael V.

Whitaker, Justine

Whitledge, Terry E.

Wick, Gary A.

Wiczyński, Grzegorz

Williams, Hannah

Wilson, Philip A.

Windt, Christian

Winters, Katherine E.

Wiora, Alicja

Wittine, Karlo

Włodek, Tomasz

Wojtaszek, Henryk

Wojtkowska, Małgorzata

Wołejsza, Piotr

Wolny, Jennifer

Woltjer, Rogier

Won, Deokhee

Won, Myoung-Soo

Wongpan, Pat

Wood, Ray

Woodward, Michael

Wortham, Jennifer L.

Wright, L. D.

Wróbel, Krzysztof

$\mathrm{Wu}$, Chensheng

Wu, Guan-James

Wu, Lianhui

$\mathrm{Wu}$, Lichuan

$\mathrm{Wu}$, Xiaoliang

$\mathrm{Wu}$, Yongsheng

Xiao, Shaoping

Xiao, Xuesu

Ximenes, Arimatéa

Xing, Yangang

Xiong, Kai

Xiros, Nikolaos

$\mathrm{Xu}$, Jiaqi

$\mathrm{Xu}$, Jiawen

$\mathrm{Xu}$, Yuhao

Yaguchi, Hiroyuki

Yahalom, Asher
Yakirevich, Alexander

Yakushev, Vladimir

Yamaguchi, Atsushi

Yamamoto, Yoshimichi

Yamazaki, Hidekatsu

Yamazaki, Tetsuo

Yan, Shiqiang

Yan, Xiaohui

Yang, Chan-Su

Yang, $\mathrm{Hu}$

Yang, Kewei

Yang, Shun-Han

Yang, Wenxian

Yang, Zhaoqing

Yaningsih, Indri

Yasuhara, Hideaki

Yasuhiro, Yoshikawa

Yasukawa, Shinsuke

Ye, Fei

Yeh, Harry

Yermolaev, Oleg Petrovich

Yi, Liang

Yim, Jeong-Bin

Yim, Unhyuk

Yokoyama, Katsuhide

Yoshida, Keiichiro

Yoshida, Sanichiro

Yoshida, Shigeo

Yoshida, Takero

Young, Ian

Yuan, Jing

Yuan, Zhiming

Yuen, Anthony Chun Yin

Yuhi, Masatoshi

Yum, Kevin Koosup

Yurchenko, Andrey A.

Zaccone, Raphael

Zachert, Hauke

Zafirakou, Antigoni

Žagar, Dejan

Zagar, Dusan

Zago, Vanessa

Zaitsev, Andrey

Zakowski, Krzysztof

Zalesny, Vladimir

Zalewski, Paweł

Zamani, Behnam

Zanforlin, Stefania

Zanuttigh, Barbara

Zarzuelo, Carmen

Zastempowski, Marcin 
Zaucha, Jacek

Zavala, Olmo

Zec, Damir

Zehfuß, Jochen

Zeleňáková, Martina

Zeng, Fanzong

Zeriouh, Ouassim

Zgrundo, Aleksandra

Zhan, Siyuan

Zhang, Fan

Zhang, Jiaying

Zhang, Puyang

Zhang, Runsen

Zhang, Wenyan

Zhang, Xuyang

Zhang, Y. Joseph

Zhang, Yanwu

Zheng, Siming

Zhou, Guangjie
Zhou, Mingxi

Zhou, Peiyuan

Zhou, Zheyu

Zhu, Hongzhong

Zhu, Ling

Zhu, Qiming

Zhu, Xiaowei

Zhukov, Igor

Zia, Muhammad Yousuf Irfan

Zidane, Ali

Zieger, Silvia E.

Zimmermann, Robert

Zis, Thalis

Zitti, Gianluca

Żółtowski, Krzysztof

Zou, Shangyan

Zukowski, Witold

Zuskin, Srdjan

Zygouri, V. 